\title{
Constrained-Induced Dysarthria Therapy: Case Report
}

\author{
Stijn Roggeman, $\mathrm{MD}^{1}$, Chris Truyers, $\mathrm{MD}^{1}$, Iwona Safin, $\mathrm{MD}^{1}$, Eline Huysman, $\mathrm{MSc}^{1}$, \\ Bernard Dan, MD, PhD ${ }^{1,2}$
}

${ }^{1}$ Stroke Rehabilitation Unit, Inkendaal Rehabilitation Hospital, Vlezenbeek;

${ }^{2}$ Université libre de Bruxelles (ULB), Bruxelles, Belgium

We describe a 33-year-old woman with chronic bulbar dysarthria after ischemic brainstem stroke who underwent a new form of constraint-induced therapy, namely constraint-induced dysarthria therapy, based on three principles: avoidance of supportive devices, intensive therapy, and gradually augmenting difficulty. After a 2-month intervention, improvement was noted for speech intelligibility, fluency, and intensity. This led to increased communicative participation, including during conversation situations, which has been maintained over a 12-month follow-up.

Keywords Dysarthria, Stroke, Constraint-induced therapy

\section{INTRODUCTION}

Speech and language difficulties such as dysarthria or aphasia are frequently seen, in addition to motor impairments in stroke patients. Among specific training programs attempting to target brain plasticity [1], constraintinduced therapy (CIT) is one of the most used strategies purposed at avoidance of 'learned nonuse', particularly constraint-induced movement therapy (CIMT) focusing on upper extremity motor dysfunction $[2,3]$. Recently, CIT was also adapted for aphasia rehabilitation, and several studies have shown positive effects of constraintinduced aphasia therapy (CIAT), but not as clear as for
CIMT [3-6]. To date, however, no type of CIT has been described for patients with dysarthria after stroke. Here, we present a patient with chronic dysarthria treated with what we called constraint-induced dysarthria therapy (CIDT).

\section{CASE REPORT}

A 33-year-old Dutch-speaking woman was admitted to our rehabilitation hospital for 2 years of multidisciplinary rehabilitation, after ischemic brainstem stroke caused by right vertebral artery dissection, presenting with lockedin syndrome. Clinically, the patient was completely de-

Received January 30, 2018; Accepted May 10, 2018

Corresponding author: Bernard Dan

Inkendaal Rehabilitation Hospital, 1 Inkendaalstraat, 1602 Vlezenbeek, Belgium. Tel: +32-25315111, Fax: +32-25324308, E-mail: bernard.dan@ulb. ac.be

ORCID: Stijn Roggeman (http://orcid.org/0000-0001-8095-0839); Chris Truyers (http://orcid.org/0000-0003-0598-0477); Iwona Safin (http://orcid. org/0000-0003-4547-7892); Eline Huysman (http://orcid.org/0000-0001-5545-3865); Bernard Dan (http://orcid.org/0000-0002-2051-9876).

(c) This is an open-access article distributed under the terms of the Creative Commons Attribution Non-Commercial License (http://creativecommons.org/ licenses/by-nc/4.0) which permits unrestricted noncommercial use, distribution, and reproduction in any medium, provided the original work is properly cited. Copyright (C) 2019 by Korean Academy of Rehabilitation Medicine 
pendent for all activities of daily living. The patient had spastic quadriplegia, dysphagia, and anarthria. Feeding was administered through a percutaneous endoscopic gastrostomy. On admission, the patient communicated using head movements: looking up for 'yes'; slightly shaking the head meant 'no'. Rehabilitation objectives included improvement of her autonomy, feeding, and speech ability.

Over 18 months of rehabilitation including conventional physical, occupational, speech therapy, and clinical psychology, we recorded slow but steady improvement of a limited number of functions. The risk of aspiration decreased sufficiently for administering oral food of adapted texture, and removing the gastrostomy. The patient regained some right upper extremity motor function, including gripping, writing, and typing. The patient used her left upper extremity only for fixating objects. The patient stayed dependent on others, for activities of daily living, including getting dressed. Communication improved partially, and also occurred non-verbally, and with supportive tools. However, no further progress was noted for the next 6 months. The following problems were observed: articulatory muscle weakness (tongue, jaw, lips, throat), and impaired respiration-phonation coordination, breathing control, speech fluency, and prosody. Persistent use of supportive communication tools was observed, namely letter chart, smartphone, tablet, and there was no improvement in speech intelligibility. So, we proposed a change in her therapy plan to improve speech through reduction of compensatory communication. In agreement with the patient and family, we administered therapy based on principles of CIT (CIMT and CIAT), which we refer to as CIDT. Protocol of CIDT was based on three principles: (1) constraint, meaning that the patient must be strongly encouraged to use verbal communication, and avoid compensations; (2) massed practice, including a defined period of intervention (10 workdays), delivered several hours a day (3-6 hours); and (3) gradual increase of the difficulty of required tasks according to the patient's functional performance [2-6].

In the presented case, during a 2-month period January-March 2017, the patient had no access to the letter chart or communication devices from awakening until 18:00, and caregivers (physicians, nurses, therapists) stimulated verbal communication. The patient received individual speech therapy (3-6 hours for 2 weeks) fo- cused on breathing, articulation, phonation, and resonance to improve intelligibility. Evaluation was made using the Dutch Speech Intelligibility Assessment [7] at the beginning and end of CIDT. We noted improvement of speech intelligibility, most effective at sentence level (Table 1), speech fluency, and intensity (increase from 64 $\mathrm{dB}$ to $75 \mathrm{~dB}$ ) [8]. On participation level, the patient was actively engaged in life situations to her satisfaction, including conversations. This outcome was maintained 12 months after discharge.

\section{DISCUSSION}

Dysarthria is a common disabling motor consequence of stroke, and it necessitates specific therapeutic attention. Speech therapy is crucial and has proven to be beneficial, in achieving functional improvement among patients after a stroke. However, evidence is not as clear as in therapies for limb motor impairments [3-6]. In this case, we administered a new intensive therapy, and found benefits regarding speech intelligibility, fluency, intensity, and patient satisfaction. To our knowledge, this is the first report of CIDT based on the three guiding principles of CIT: intensive, constraint, and shaping $[2,3]$. Concerning beneficial effects of CIT, CIMT (original and modified) has proven to be one of the most effective interventions for the upper paretic limb after a stroke [2].

Table 1. Assessment results

\begin{tabular}{llc}
\hline \multicolumn{1}{c}{ Assessment } & Prior to CIDT & After CIDT \\
\hline NSVO & & \\
\hline First consonant & $6 / 19$ & $6 / 19$ \\
\hline Last consonant & $10 / 15$ & $12 / 15$ \\
Middle vocal & $14 / 16$ & $13 / 16$ \\
\hline Total & $30 / 50(60)$ & $31 / 50(62)$ \\
NSVO-Z & & \\
\hline $\begin{array}{l}\text { Total number of correctly } \\
\text { intelligible words }\end{array}$ & $56 / 120(46.7)$ & $77 / 120(64.2)$ \\
\hline \begin{tabular}{l} 
Intensity (dB) \\
\hline
\end{tabular} & 64 & 75 \\
\hline
\end{tabular}

Values are presented as number (\%).

CIDT, constraint-induced dysarthria therapy; NSVO, Nederlandstalig Spraakverstaanbaarheidsonderzoek (Dutch Speech Intelligibility Assessement); NSVO-Z, ederlandstalig Spraakverstaanbaarheidsonderzoek - Zinsniveau (Dutch Speech Intelligibility Assessement - Sentence level). 
It can result in meaningful effect on arm-hand activities, and amount and quality of arm-hand use, in specific and basic activities of daily living [2]. CIAT seems to be successful as treatment strategy in aphasia rehabilitation, but current evidence is not as clear as for CIMT [3]. It was found that 14 hours of CIAT for 2 weeks combining individual and group sessions, was the most efficient strategy and reached similar results as 30 hours of group therapy, either with or without constraint [3]. Massed practice is likely to be the most useful component of CIAT, while the role of constraint must be further examined [3]. Social interactions, e.g., with a group or the family, seems to be a component to consider when applying CIAT [3-5].

Mechanisms underlying improvement by CIT are poorly understood. Enhanced cortical neuroplasticity, documented by transcranial magnetic stimulation and fMRI in subacute and chronic phase, may be associated with learned nonuse and the ability for compensation, rather than neurological repair or recovery $[3,9]$. As regards the acute phase, improvement is more clearly characterized by increased levels of neuroplasticity [3]. This is the case for CIMT, whereas CIAT seems not to be associated with significant changes in cortical activity [9].

In our case, the CIDT led to improvement of speech intelligibility, most effective for sentences, which best convey linguistic-situational information of speech [10]. CIDT was not administered during the period conventionally used in other CIT paradigms because progress, particularly at the level of sentences, was observed during the course of therapy. So, we decided with the patient to pursue therapy until communication ability was stable. Another limitation of the study is the single-case description, rather than group or case-control design.

In conclusion, intensive speech therapy in the form of CIDT may be a beneficial rehabilitation approach as shown by our case. Its implementation in neurological rehabilitation must be further investigated, with particular attention to inclusion criteria, dosage, and effectiveness.

\section{CONFLICT OF INTEREST}

No potential conflict of interest relevant to this article was reported.

\section{REFERENCES}

1. Belardinelli P, Laer L, Ortiz E, Braun C, Gharabaghi A. Plasticity of premotor cortico-muscular coherence in severely impaired stroke patients with hand paralysis. Neuroimage Clin 2017;14:726-33.

2. Kwakkel G, Veerbeek JM, van Wegen EE, Wolf SL. Constraint-induced movement therapy after stroke. Lancet Neurol 2015;14:224-34.

3. Woldag H, Voigt N, Bley M, Hummelsheim H. Constraint-induced aphasia therapy in the acute stage: what is the key factor for efficacy? A randomized controlled study. Neurorehabil Neural Repair 2017;31:7280.

4. Szaflarski JP, Ball AL, Vannest J, Dietz AR, Allendorfer JB, Martin AN, et al. Constraint-induced aphasia therapy for treatment of chronic post-stroke aphasia: a randomized, blinded, controlled pilot trial. Med Sci Monit 2015;21:2861-9.

5. Zhang J, Yu J, Bao Y, Xie Q, Xu Y, Zhang J, Wang P. Constraint-induced aphasia therapy in post-stroke aphasia rehabilitation: a systematic review and metaanalysis of randomized controlled trials. PLoS One 2017;12:e0183349.

6. Ciccone N, West D, Cream A, Cartwright J, Rai T, Granger A, et al. Constraint-induced aphasia therapy (CIAT): a randomised controlled trial in very early stroke rehabilitation. Aphasiology 2016;30:566-84.

7. Martens H, Van Nuffelen G, De Bodt M. NSVO-Z: Nederlandstalig Spraakverstaanbaarheidsonderzoek Zinsniveau [Dutch Speech Intelligibility Assessment]. Herentals, Belgium: Vlaamse Vereniging voor Logopedisten (VVL); 2010.

8. Maryn Y, Weenink D. Objective dysphonia measures in the program Praat: smoothed cepstral peak prominence and acoustic voice quality index. J Voice 2015;29:3543.

9. Nenert R, Allendorfer JB, Martin AM, Banks C, Ball A, Vannest J, et al. Neuroimaging correlates of poststroke aphasia rehabilitation in a pilot randomized trial of constraint-induced aphasia therapy. Med Sci Monit 2017;23:3489-507.

10. Kalikow DN, Stevens KN, Elliott LL. Development of a test of speech intelligibility in noise using sentence materials with controlled word predictability. J Acoust Soc Am 1977;61:1337-51. 